\title{
Tesis en formato de artículo científico: oportunidad para incrementar la producción científica universitaria
}

\section{Thesis in scientific paper format: opportunity to increase scientific production in Peruvian universities}

Correspondencia Percy Mayta-Tristán p.mayta@gmail.com

Citar como: Mayta-Tristán P Tesis en formato de artículo cientifico: oportunidad para incrementar la producción científica universitaria. Acta Med Peru. 2016;33(2):95-8
Percy Mayta-Tristán ${ }^{1,2}$

1 Escuela de Medicina, Universidad Peruana de Ciencias Aplicadas. Lima, Perú.

2 Acta Médica Peruana, Colegio Médico del Perú. Lima, Perú.

La producción científica peruana en salud es baja, concentrada en la capital y sólo dos de las 30 universidades que imparten la carrera de medicina producen más de un artículo por año en el Science Citation Index en el periodo 2000-2009 [1]. Tradicionalmente se menciona que la tesis es la primera oportunidad de hacer una investigación; sin embargo, en aquellas universidades que tienen varias opciones de titulación solo $11 \%$ de internos refiere tener la intención de hacer tesis [2], entre los principales factores referidos para no hacer tesis figuran los trámites engorrosos, escaso apoyo de la facultad, no pertenecer a una sociedad científica estudiantil, falta de tiempo o interés, entre otros [3,4]. Por otro lado, solo 16/32 escuelas de medicina evaluadas en el 2011 tenían a la tesis como requisito obligatorio para obtener su título [5] y recientemente se ha quitado el requisito de hacer tesis para obtener el título de especialista después del residentado médico [6].

Hacer una tesis demanda un esfuerzo que si bien recae sobre el tesista, debe haber un compromiso de su universidad en brindarle la asesoría respectiva, por ejemplo, la nueva Ley Universitaria $N^{\circ}$ 30220 del año 2014 en su artículo 100.13 menciona "el alumno tiene el derecho de gratuidad para el asesoramiento de su tesis, para obtener el grado de Bachiller, por una sola vez" [7]. Sin embargo, esto no siempre sucede así, y en muchos casos, los asesores suelen ser una traba, además de los trámites administrativos para realizar la tesis [8]. ¿Por qué? Porque la tesis como está concebida no le suma al asesor ni a la universidad, incluso puede ser una fuente de desprestigio al evidenciarse una alta frecuencia de plagio dentro de los marcos teóricos [9].

Ante ello, algunas universidades han desarrollado una propuesta distinta en relación al formato de cómo hacer la tesis. En vez de ser un documento extenso de más de 100 páginas con una gran cantidad de figuras y variables muchas veces repetitivos y que se quedan en las bibliotecas, cambiaron sus normativas para que esta se redacte en el formato de un artículo científico, e incluso se envíe a publicar a una revista indizada. Teniendo como primera premisa de que las tesis son trabajos de investigación y como tales deberían poder ser publicadas en revistas científicas indizadas.

La pionera fue la Escuela de Medicina de la Universidad Peruana Cayetano Heredia (UPCH), que a través de diversos estudios han podido demostrar el impacto de esta medida. Cuando se hacían tesis tradicionales solo el $17,6 \%$ de éstas lograban ser publicadas [10], frecuencia que incrementó al 22,4\% cuando se dispuso el cambio de formato al de un artículo científico, y luego subió al 40,3\% cuando se implementó el requisito de envío a una revista indizada en SciELO o en bases de datos superiores como MEDLINE, Scopus [11]. 
Tabla 1. Modalidad alternativa de tesis en formato de artículo científico*.

\begin{tabular}{|c|c|c|c|}
\hline Características & Título profesional & $\begin{array}{l}\text { Maestría / Segunda } \\
\text { especialidad }\end{array}$ & Doctorado \\
\hline \multicolumn{4}{|l|}{ Autoria filiación } \\
\hline Número de tesistas & Uno o dos & Uno & Uno \\
\hline $\begin{array}{l}\text { Posición en orden de autoría / } \\
\text { Corresponsal }\end{array}$ & Primeros & Primero & Primero o corresponsal \\
\hline $\begin{array}{l}\text { Forma de incluir Afiliación } \\
\text { institucional }\end{array}$ & Facultad, Universidad, Ciudad, País & $\begin{array}{l}\text { Facultad/Escuela de Posgrado, } \\
\text { Universidad, Ciudad, País }\end{array}$ & $\begin{array}{l}\text { Facultad / Escuela de Posgrado, } \\
\text { Universidad, Ciudad, País }\end{array}$ \\
\hline Afiliación institucional exclusiva & $\mathrm{Si}$ & No & No \\
\hline \multicolumn{4}{|l|}{ De los artículos } \\
\hline Número de artículos & Uno & Uno & Tres \\
\hline $\begin{array}{l}\text { Publicación/aceptación del } \\
\text { artículo }\end{array}$ & $\begin{array}{l}\text { No necesario, solo confirmación de } \\
\text { envío }\end{array}$ & Sí & Sí \\
\hline Tipo de artículo & $\begin{array}{l}\text { Un articulo original o de investigación, } \\
\text { no aceptable otra modalidad } \\
\text { (revisiones de tema, ensayos, } \\
\text { originales, comunicaciones cortas, } \\
\text { editoriales, cartas al editor u otras). }\end{array}$ & $\begin{array}{l}\text { Un artículo original o de investigación, } \\
\text { no aceptable otra modalidad } \\
\text { (revisiones de tema, ensayos, } \\
\text { originales, comunicaciones cortas, } \\
\text { editoriales, cartas al editor u otras). }\end{array}$ & $\begin{array}{l}\text { Tres artículos originales o de } \\
\text { investigación, no aceptable otra } \\
\text { modalidad (revisiones de tema, ensayos, } \\
\text { originales, comunicaciones cortas, } \\
\text { editoriales, cartas al editor u otras). }\end{array}$ \\
\hline Nivel de indización de la revista & $\begin{array}{l}\text { Según disponga la universidad o } \\
\text { programa académico }\end{array}$ & Scopus o ISI & $\begin{array}{l}\text { Scopus o ISI, al menos uno en cuartil } \\
\mathrm{O}_{1}-\mathrm{Q}_{2}\end{array}$ \\
\hline Publicación en inglés & No necesario & No necesario & Al menos uno \\
\hline \multicolumn{4}{|l|}{ Contenido del documento } \\
\hline Artículo enviado & Sí & Sí & Sí \\
\hline Peer review & No necesario & Sí & Sí \\
\hline Versión publicada/aceptada & No necesario & Sí & Sí \\
\hline Texto introductorio** & No & No & Sí \\
\hline
\end{tabular}

* Son requisitos básicos, cada Escuela o Programa de posgrado puede incrementar los requisitos acorde a los objetivos del programa.

** Texto que precede a los tres artículos que los contextualiza sobre el problema de investigación abordado.

La Escuela de Medicina de la Universidad Santo Toribio de Mogrovejo en Chiclayo también implementó esta modalidad, pero con el requisito de enviar a revistas indizadas en LILACS o bases de datos superiores, y su porcentaje de tesis publicadas llega al $30 \%$ [12]. Finalmente, la Escuela de Medicina de la Universidad Peruana de Ciencias Aplicadas lo implementó en su reglamento de grados y títulos [13] pero con el requisito de que la revista esté indizada en Scopus/Medline donde los tesistas (hasta tres) sean primeros autores, en las dos primeras promociones de egresados más del $50 \%$ de las tesis han sido publicadas antes de los dos años de sustentadas (Mayta-Tristán, comunicación personal).

En el caso de maestría, la Escuela de Postgrado de la UPCH implementó esta modalidad, donde el requisito es que el artículo sea publicado en una revista indizada en MEDLINE o una base de datos superior como SCOPUS o Science Citation Index [14]. En el caso de la Universidad Nacional Mayor de San Marcos, existe la exigencia de que el alumno acredite la aceptación o publicación de un artículo en una revista indizada y que deba ser parte de la tesis tanto para el grado de magíster como doctor [15]. Finalmente, hay la iniciativa de la Escuela de Postgrado de la Universidad Privada Antenor Orrego de implementar que la tesis sea la suma de tres artículos publicados en revistas Scopus, donde el tesistas es el primer autor o corresponsal en los tres artículos, los cuales deben estar vinculados (Jose Caballero, comunicación personal).

La nueva Ley Universitaria y su proceso de reglamentación por la Superintendencia Nacional de Educación Superior Universitaria (SUNEDU), crea una oportunidad valiosa para poder implementar en sus definiciones la posibilidad de que la tesis pueda hacerse en formato de artículo científico. En ese contexto, se plantean las siguientes propuestas para el nuevo reglamento de grados y títulos que viene desarrollando SUNEDU y que fueron enviadas a esa institución.

Trabajo de investigación: Es un tipo de trabajo académico, el cual tiene un propósito claramente definido, responde a una pregunta de investigación, se apoya en conocimiento existente, aplica el método científico, aporta evidencia verificable, proporciona explicaciones objetivas y racionales, contextualiza sus hallazgos y mantiene un espíritu autocrítico. Es de carácter público y como tal, está sujeto a debate. Tiene un asesor y puede ser individual o grupal hasta cinco personas.

Tesis: es un trabajo de investigación que pasa por sustentación pública y la aprobación de un jurado. Puede ser presentado hasta por dos tesistas para el Título Profesional y Segunda 
Tabla 2. Análisis del proceso tradicional de graduación por tesis y la opción de tesis en formato de artículo científico.

Tesis tradicional

El alumno egresa con una tesis en su curriculum.

Algunas tesis se publican en revistas indizadas.

En el caso de que se publiquen las investigaciones desarrolladas como tesis contribuyen a la producción científica de la universidad y el país.

El proceso de evaluación de calidad está dado solo por profesores de la universidad.

Hay poca motivación de los asesores en apoyar las tesis.

El alumno no culmina el proceso de investigación al faltarle la experiencia de publicación.

El alumno no ha tenido la experiencia curricular de seleccionar una revista, enviar el artículo, hacer seguimiento y responder observaciones de los pares.

Las investigaciones sólo son visibles desde las bibliotecas de la universidad, o en el mejor caso desde repositorios electrónicos.

Las revistas peruanas buscan a los tesistas para que publiquen sus investigaciones.

\section{Tesis en formato artículo cientifico}

El alumno egresa con un artículo publicado y una tesis en su curriculum.

Todas (maestría / doctorado) o la mayoría (título) las tesis son publicadas en revistas indizadas.

Todas las investigaciones desarrolladas como tesis bajo esta modalidad contribuyen a la producción científica de la universidad y el país.

El proceso de evaluación de calidad está dado por los profesores de la universidad y por la revisión por pares que realiza la revista científica donde se publica el artículo.

Los asesores tienen la motivación de que la tesis será publicada y serían autores si cumplen los criterios de autoría. El alumno aprende el proceso de investigación completo: desde la idea hasta la publicación.

El alumno aprende como seleccionar una revista, enviar el artículo, hacer seguimiento y responder observaciones de los pares.

Las investigaciones son visibles desde las revistas donde están publicadas y en las bases de datos que estén indizadas (p.e. ISI, Scopus, MEDLINE, SciELO, etc).

Los tesistas buscan a las revistas peruanas e internacionales para publicar sus tesis.
Especialidad, y solo por uno para los grados de Magístery Doctor. El formato de presentación puede ser el tradicional que depende del campo de conocimiento, universidad y programa académico, o bajo la modalidad de artículo científico, el cual difiere según el grado académico como se describe a continuación (Tabla 1):

- Título profesional y segunda especialidad. La tesis, que corresponde a un trabajo de investigación, se presenta en formato de artículo científico de una revista indizada en la base de datos a determinar por la Universidad o Programa Académico, el cual debe haber sido enviado a publicar. El o los tesistas (hasta dos) deben ser los primeros autores con afiliación institucional de su universidad. El documento incluye el artículo enviado a publicar, la constancia de recepción de la revista y un compromiso del seguimiento de publicación por parte del(los) tesista(s) y asesor en caso no se haya publicado aún.

- Magíster. La tesis, que corresponde a un trabajo de investigación, es un artículo publicado en una revista indizada en Scopus, Science Citation Index ( $\mathrm{SCl}$ ) o una base de datos específica del campo de conocimiento determinada por la universidad o programa académico (p.e. Medline, Psylndex, etc). Solo es presentada por un tesista, el cual debe ser el primer autor de la publicación, con afiliación de su universidad. El documento de la tesis incluye el artículo publicado, el proceso de revisión por pares (observaciones, respuestas y correcciones a los revisores de la revista) y la versión del artículo enviado.
- Doctor. La tesis es la suma de tres (o más) artículos publicados en revistas indizadas en Scopus o en el Science Citation Index $(\mathrm{SCl})$, estos artículos deben estar vinculados entre sí, los tres son trabajos de investigación, al menos uno de ellos publicado en inglés, y al menos uno de ellos en una revista de cuartil 102 de Scopus o SCl. El documento de tesis incluye un texto introductorio que contextualiza el problema de investigación abordado por los tres artículos, los tres artículos publicados, y los procesos de revisión seguidos en las respectivas revistas. El tesista debe ser el primer autor o corresponsal en todos los artículos, y debe tener la afiliación institucional de su universidad.

En la tabla 2 se resume algunas de las ventajas de implementar esta modalidad, la cual no reemplaza a la tesis tradicional sino que es una alternativa que puede incrementar la producción científica de las universidades, y que junto a los recientes mecanismos de incentivos a las publicaciones como viene haciendo CONCYTEC [16], ayudarán a las revistas científicas nacionales a mejorar la calidad y cantidad de lo que publican, que a futuro redundará en más revistas indizadas en bases como Medline y Scopus, que es lo que buscamos en el equipo editorial de Acta Médica Peruana [17]

\section{Agradecimientos}

A todos los investigadores que han revisado y contribuido a mejorar esta propuesta que fue remitida a SUNEDU. 


\section{REFERENCIAS BIBLIOGRÁFICAS}

1. Huamaní C, Mayta-Tristán P. Producción científica peruana en medicina y redes de colaboración, análisis del Science Citation Index 2000-2009. Rev Peru Med Exp Salud Publica. 2010;27(3):315-25.

2. Galán-RodasE, Diaz-VélezC, Mejia CR. Intención en la modalidad de titulación de internos de medicina en el Perú, 2010. Rev Peru Med Exp Salud Publica. 2011;28(2):390-9.

3. Ramos-Rodríguez Ml, Sotomayor R. Realizar o no una tesis: razones de estudiantes de medicina de una universidad pública y factores asociados. Rev Peru Med Exp Salud Publica. 2008;25(3):322-24.

4. Alarco JJ, Aguirre-Cuadros E, Aliaga-Chávez Y, Álvarez-Andrade EV. Factores asociados a la realización de tesis en pregrado de medicina en una universidad pública del Perú. CIMEL. 2010;15(2):66-70.

5. Mayta-Tristan . Características de los cursos de investigación en escuelas de medicina del Perú. Arch Med. 2015;1182):e1.

6. Valle R, Perales A. Nueva normativa de titulación en el residentado médico en el Perú: problemas y perspectivas. Rev Peru Med Exp Salud Publica. 2016;32(2):357-61.

7. Perú, Congreso de la República. Ley Nº 30220: Ley Universitaria. Lima: Congreso de la República; 2014.

8. Mejia CR, Inga-Berrospi F, Mayta-Tristán P.Titulación por tesis en escuelas de medicina de Lima, 2011: características, motivaciones y percepciones. Rev Peru Med Exp Salud Publica. 2014;31(3):50914 .

9. Saldaña-Gastulo JJC, Quezada-Osoria CC, Peña-Oscuvilca A, Mayta-Tristán P. Alta frecuencia de plagio en tesis de medicina de una universidad pública peruana. Rev Peru Med Exp Salud Publica. 2010;27(1):63-7.

10. Arriola-Quiroz I, Curioso WH, Cruz-Encarnación M, Gayoso O. Characteristics and publication patterns of theses from a Peruvian medical School. Heath Info Libr J. 2010;27(2):148-54.
11. Ticse R, Chavez M, Levano C, Sanchez C, Samalvides F. Impacto de una intervención universitaria en la frecuencia de publicaciones generadas de los trabajos de investigación sustentados para la titulación de médico cirujano de los alumnos egresados de la Universidad Peruana Cayetano Heredia durante el periodo 20052010. En: IV Congreso Peruano de Educación Médico. Lima, Perú: Asociación Peruana de Facultades de Medicina; 2013.

12. Leon Jimenez F. Pregrado en medicina humana: ¿es posible graduarse publicando? Rev Med Hered. 2013;24(2):175-77.

13. Universidad Peruana de Ciencias Aplicadas. Reglamento de Grados y Títulos de la Facultad de Ciencias de la Salud [Internet]. Lima; UPC; 2013 [citado el 30 de mayo de 2016]. Disponible en: http://www.upc.edu.pe/ sites/default/files/upc/home/sub_secciones/es/politicas_reglamentos_y_ normas/pagina_primaria_descargas/sica-reg-07_reglamento_grados_y_ titulos_de_la_facultad_de_ciencias_de_la_salud.pdf

14. Escuela de Postgrado, Universidad Peruana Cayetano Heredia. Graduación de maestría mediante la modalidad de publicación internacional [Internet]. Lima: UPCH; 2011. [citado el 30 de mayo de 2016]. Disponible en: http://www.upch.edu.pe/epgvac/public/doc/ graduacion_por_articulo.pdf

15. Universidad Nacional Mayor de San Marcos. Reglamento general de estudios de postgrado [Internet]. Lima: UNMSM; 2009 [citado el 30 de mayo de 2016]. Disponible en: http://upg.medicina.unmsm.edu. pe/images/Postgrado/REGLAMENTO.pdf

16. Consejo Nacional de Ciencia, Tecnología e Innovación Tecnológica. Incentivo para la publicación efectiva de artículos científicos en revistas indizadas - 2015 [Internet]. Lima: Concytec; 2015 [citado el 30 de mayo de 2016]. Disponible en: http://www.cienciactiva.gob. pe/cienciactiva/convocatorias/premios/estimulo-articulo-cientificos

17. Zolezzi A, Mayta-Tristán P. Sentando las bases para la indización: cambios en las instrucciones para los autores de Acta Médica Peruana. Acta Med Peru. 2016;26(1):7-8.

\title{
Las ediciones anteriores de Acta Médica Peruana están disponibles en:
}

\author{
www.redalyc.org
}

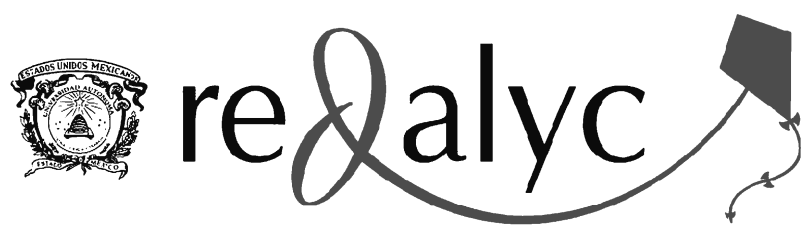

